Glasgow Math. J. 48 (2006) 533-545. (C) 2006 Glasgow Mathematical Journal Trust. doi:10.1017/S0017089506003260. Printed in the United Kingdom

\title{
DUO MODULES
}

\author{
A. Ç. ÖZCAN, A. HARMANCI \\ Hacettepe University, Department of Mathematics 06532 Beytepe, Ankara TURKEY \\ e-mail: ozcan@hacettepe.edu.tr,harmanci@hacettepe.edu.tr \\ and P. F. SMITH \\ University of Glasgow Department of Mathematics Glasgow G12 8QW Scotland, UK \\ e-mail:pfs@maths.gla.ac.uk
}

(Received 24 February, 2006; accepted 31 July, 2006)

\begin{abstract}
Let $R$ be a ring. An $R$-module $M$ is called a (weak) duo module provided every (direct summand) submodule of $M$ is fully invariant. It is proved that if $R$ is a commutative domain with field of fractions $K$ then a torsion-free uniform $R$-module is a duo module if and only if every element $k$ in $K$ such that $k M$ is contained in $M$ belongs to $R$. Moreover every non-zero finitely generated torsion-free duo $R$-module is uniform. In addition, if $R$ is a Dedekind domain then a torsion $R$-module is a duo module if and only if it is a weak duo module and this occurs precisely when the $P$-primary component of $M$ is uniform for every maximal ideal $P$ of $R$.
\end{abstract}

2000 Mathematics Subject Classification. 16D99, 13C12 (13B20).

1. Introduction. Throughout all rings have an identity and all modules are unital. $R$ is a ring and $M$ a right $R$-module. A submodule $N$ of $M$ is called fully invariant if $f(N)$ is contained in $N$ for every $R$-endomorphism $f$ of $M$. Let $S=\operatorname{End}\left(M_{R}\right)$, the ring of $R$-endomorphisms of $M$. Then $M$ is a left $S$-, right $R$-bimodule and a submodule $N$ of the right $R$-module $M$ is fully invariant if and only if $N$ is a sub-bimodule of $M$. Clearly 0 and $M$ are fully invariant submodules of $M$. The right $R$-module $M$ is called a duo module provided every submodule of $M$ is fully invariant. For example, if $U$ is a simple right $R$-module, then clearly $U$ is a duo module but $U \oplus U$ is not duo. The ring $R$ is called a right duo ring if the right $R$-module $R$ is a duo module. Note that a ring $R$ is a right duo ring if and only if every right ideal of $R$ is a two-sided ideal; equivalently $R a$ is contained in $a R$ for every element $a$ in $R$. Clearly commutative rings and division rings are right (and left) duo rings but any $2 \times 2$ matrix ring over such a ring is not a right (or left) duo ring.

We begin with a simple observation.

Lemma 1.1. Let $R$ be any ring. Then a right $R$-module $M$ is a duo module if and only if for each endomorphism $f$ of $M$ and each element $m$ of $M$ there exists $r$ in $R$ such that $f(m)=m r$.

Proof. The necessity follows because $f(m R)$ is contained in $m R$. Conversely, note that the stated condition implies that $f(N)$ is contained in $N$ for every submodule $N$ and endomorphism $f$ of $M$. It follows that $M$ is a duo module. 
A good source of duo modules is provided by multiplication modules. Let $R$ be a commutative ring. An $R$-module $M$ is called a multiplication module provided that for each submodule $N$ of $M$ there exists an ideal $I$ of $R$ such that $N=I M$. Among examples of multiplication $R$-modules we can mention projective ideals of $R$ (see [11, Theorem 1]), ideals of $R$ generated by idempotents (see [3, Corollary 1.3]) and finitely generated $R$-modules $M$ such that every localization of $R$ with respect to a maximal ideal of $R$ is cyclic (see [3, Theorem 1.2]). Note further that if $I$ is a multiplication ideal of $R$ (i.e. $I$ is an ideal of $R$ and a multiplication $R$-module) and $M$ is a multiplication $R$-module then the $R$-module $I M$ is a multiplication $R$-module (see [3, Corollary 1.4]). A projective $R$-module $M$ is a multiplication module if and only if $M$ is a duo module (see [10, Corollary B]). Moreover, a finitely generated module $M$ with annihilator $A$ in $R$ is a multiplication module if and only if $M$ is a projective $(R / A)$-module and a duo $R$-module (see [10, Corollary $\mathrm{C}]$ ).

Let $R$ be any ring. Let $M$ be an $R$-module and let $f$ be an endomorphism of $M$. For any submodule $N$ of $M$, we set $f^{-1}(N)=\{m \in M: f(m) \in N\}$. Note that $f^{-1}(N)$ is a submodule of $M$ and that $f\left(f^{-1}(N)\right) \subseteq N$. Note further that $f\left(f^{-1}(N)\right)=N$ in case $f$ is an epimorphism. Moreover, for any submodules $L \subseteq N$ of $M$, we have $f^{-1}(L) \subseteq f^{-1}(N)$. For any positive integer $n$, we shall denote $\left(f^{n}\right)^{-1}(N)$ by $f^{-n}(N)$; i.e. $f^{-n}(N)=\left\{m \in M: f^{n}(m) \in N\right\}$.

Another source of duo modules is provided by uniserial modules. A module $M$ is called uniserial if, for all submodules $L$ and $N$ of $M$, either $L \subseteq N$ or $N \subseteq L$.

THEOREM 1.2. Let $M$ be a uniserial module which either satisfies the ascending chain condition on cyclic modules or is Artinian. Then $M$ is a duo module.

Proof. Suppose that $M$ satisfies the acc on cyclic submodules. Let $0 \neq m \in M$ and let $f$ be an endomorphism of $M$. Suppose that $f(m) \notin m R$. Then $m \in f(m) R$ and hence $m=f(m) r$ for some $r \in R$. It follows that $f^{n}(m)=f^{n+1}(m) r$ for every positive integer $n$. Thus $m R \subseteq f(m) R \subseteq f^{2}(m) R \subseteq \cdots$. By hypothesis, there exists a positive integer $k$ such that $f^{k}(m) R=f^{k+1}(m) R$. There exists $s \in R$ such that $f^{k+1}(m)=f^{k}(m) s=f^{k}(m s)$. Hence $f(m)-m s \in \operatorname{ker}\left(f^{k}\right)$. If $m R \subseteq \operatorname{ker}\left(f^{k}\right)$, then $f^{k}(m)=0$ and hence $m=f^{k}(m) r^{k}=$ 0 , a contradiction. Thus $k e r\left(f^{k}\right) \subseteq m R$ and hence $f(m)-m s \in m R$, so that $f(m) \in m R$, a contradiction. It follows that $f(m) \in m R$. By Lemma 1.1, $M$ is a duo module.

Now suppose that $M$ is Artinian. Suppose that $M$ is not a duo module. There exist a non-zero submodule $L$ of $M$ and an endomorphism $g$ of $M$ such that $g(L) \nsubseteq L$. Hence $L \subseteq g(L)$. It follows that $L \subseteq g(L) \subseteq g^{2}(L) \subseteq \cdots$. Consider the descending chain $M \supseteq g(M) \supseteq g^{2}(M) \supseteq \cdots$ of submodules of $M$. Because $M$ is Artinian, there exists a positive integer $n$ such that $g^{n}(M)=g^{n+1}(M)$, and hence $M=g(M)+\operatorname{ker}\left(g^{n}\right)$. If $g(M) \subseteq \operatorname{ker}\left(g^{n}\right)$, then $g^{n+1}(M)=0$ and hence $L \subseteq g^{n+1}(L)=0$, a contradiction. Thus $\operatorname{ker}\left(g^{n}\right) \subseteq g(M)$ so that $M=g(M)$.

If $L \subseteq g^{-1}(L)$, then $g(L) \subseteq g\left(g^{-1}(L)\right) \subseteq L$, a contradiction. Thus $g^{-1}(L) \subseteq L$. It follows that $L \supseteq g^{-1}(L) \supseteq g^{-2}(L) \supseteq \cdots$ is a descending chain of submodules of $M$. There exists a positive integer $k$ such that $g^{-k}(L)=g^{-(k+1)}(L)$. Because $g^{k+1}$ is an epimorphism, we have $g(L)=g^{k+1}\left(g^{-k}(L)\right)=g^{k+1}\left(g^{-(k+1)}(L)\right)=L$, a contradiction. Thus $M$ is a duo module.

We have already observed that the direct sum of duo modules need not be a duo module. Note the following fact.

Proposition 1.3. Any direct summand of a duo module is also a duo module. 
Proof. Let $M$ be a duo module such that $M=M^{\prime} \oplus M^{\prime \prime}$ is the direct sum of submodules $M^{\prime}$ and $M^{\prime \prime}$. Let $p: M \rightarrow M^{\prime}$ be the canonical projection and let $i: M^{\prime} \rightarrow M$ denote inclusion. Suppose that $f$ is an endomorphism of $M^{\prime}$ and $N$ is any submodule of $M^{\prime}$. Then $g=$ if $p$ is an endomorphism of $M$ and $f(N)=g(N)$, which is contained in $N$ because $M$ is duo. It follows that $M^{\prime}$ is a duo module.

In general submodules of duo modules are not duo modules, so that the next result is of some interest.

Proposition 1.4. Let $M$ be a duo module.

(i) If $M$ is quasi-injective, then every submodule of $M$ is a duo module.

(ii) If $M$ is quasi-projective, then every homomorphic image of $M$ is duo.

Proof. (i) Let $L$ be any submodule of $M$. Let $N$ be a submodule of $L$ and let $f$ be an endomorphism of $L$. Because $M$ is quasi-injective, $f$ can be lifted to an endomorphism $f^{*}$ of $M$. Then $f(N)=f^{*}(N)$, which is contained in $N$ because $M$ is a duo module. It follows that $L$ is a duo module.

(ii) Let $K$ be a submodule of $M$. Let $H$ be a submodule of $M$ containing $K$ and let $g$ be an endomorphism of the module $M / K$. Because $M$ is quasi-projective, there exists an endomorphism $g^{*}$ of $M$ such that $g(m+K)=g^{*}(m)+K$ for all $m$ in $M$. Because $M$ is a duo module, $g^{*}(H)$ is contained in $H$ and hence $g(H / K)$ is contained in $H / K$. It follows that $M / K$ is a duo module.

To see that submodules of duo modules are not duo modules consider the following example. Let $K$ be a field and let $V$ be a two-dimensional vector space over $K$. Let the ring $R$ be the trivial extension of $V$ by $K$. Thus $R$ is the $K$-vector space $K \oplus V$ and multiplication is defined in $R$ as follows: $(a, u)(b, v)=(a b, a v+b u)$ for all $a, b$ in $K$ and $u, v$ in $V$. Because $R$ is a commutative ring, the $R$-module $R$ is a duo module. However, the submodule $0 \oplus V$ is not a duo module, being the direct sum of two isomorphic simple modules. We do not know an example of a duo module $M$ and a submodule $N$ of $M$ such that $M / N$ is not a duo module.

Here is another simple observation.

Proposition 1.5. Let $M$ be a module such that every countably generated submodule is a duo module. Then $M$ is a duo module.

Proof. Let $m$ be any element of $M$ and let $f$ be any endomorphism of $M$. Let $N=m R+f(m) R+f^{2}(m) R+\cdots$. Then $N$ is a countably generated submodule of $M$ and the restriction of $f$ to $N$ is an endomorphism of $N$. By Lemma 1.1 and hypothesis, $f(m)=m r$ for some $r$ in $R$. Again by Lemma 1.1, $M$ is a duo module.

Of course, many modules are not duo modules. Note the following fact.

Proposition 1.6. Let $R$ be a proper subring of a ring $S$. Then the right $R$-module $S$ is not a duo module.

Proof. Let $s$ be any element of the ring $S$ such that $s$ does not belong to $R$. Then the mapping $f: S \rightarrow S$ defined by $f(a)=s a$, for all $a$ in $S$, is an $R$-homomorphism. Note that $s=f(1)$, so that $R$ is not a fully invariant submodule of the $R$ module $S$. 
Next we consider some simple properties of duo modules. Let $R$ be a ring. An $R$ module $M$ is called Hopfian provided that every surjective endomorphism of $M$ is an isomorphism. For example, every Noetherian module is Hopfian (see [1, Lemma 11.6]). The module $M$ is called generalized Hopfian if every surjective endomorphism of $M$ has a small kernel (see [4]). On the other hand, the module $M$ is called co-Hopfian if every injective endomorphism is an isomorphism. By [1, Lemma 11.6], Artinian modules are co-Hopfian. Next, in [5], the module $M$ is called weakly co-Hopfian if every injective endomorphism has an essential image.

Proposition 1.7. Every duo module is a generalized Hopfian and a weakly coHopfian module.

Proof. Let $f$ be any surjective endomorphism of $M$. Let $L$ be any submodule of $M$ such that $M=k e r f+L$. Then $M=f(M)=f(k e r f+L)=f(L)$, which is contained in $L$. It follows that kerf is a small submodule of $M$. Next let $g$ be an injective endomorphism of $M$. Let $N$ be any submodule of $M$ such that $N \cap g(M)=0$. Because $N$ is fully invariant, we obtain $g(N)=0$ and hence $N=0$. It follows that $g(N)$ is an essential submodule of $M$.

In contrast to Proposition 1.7, duo modules need not be Hopfian or co-Hopfian in general. Moreover, Hopfian modules need not be duo and neither need co-Hopfian modules be duo. For example, let $R$ denote the ring $\mathbb{Z}$ of integers. For any prime $p$, the Prüfer $p$-group $\mathbb{Z}\left(p^{\infty}\right)$ is an Artinian uniserial $R$-module and hence is a duo module (Theorem 1.2) but the mapping $f: \mathbb{Z}\left(p^{\infty}\right) \rightarrow \mathbb{Z}\left(p^{\infty}\right)$ defined by $f(x)=p x$ for all $x$ in $\mathbb{Z}\left(p^{\infty}\right)$ is a surjective endomorphism which is not an isomorphism. Moreover, the $R$-module $R$ is a duo module but the mapping $g: R \rightarrow R$ defined by $g(a)=2 a$, for all $a$ in $R$, is an injective endomorphism which is not an isomorphism.

Let $R$ be a ring and let $M$ be a right $R$-module with endomorphism ring $S$. It is easy to check that every idempotent of $S$ is central in $S$ if and only if every direct summand of $M$ is fully invariant, and modules $M$ with this property will be called weak duo modules. It is proved in [2] that any weak duo module which satisfies the finite exchange property satisfies the (unrestricted) exchange property.

It is easy to give examples of weak duo modules. Clearly any indecomposable module is a weak duo module, so that in particular any uniform module is a weak duo module. If $R$ is a domain, then the right $R$-module $R$ is indecomposable and hence a weak duo module. However, if the right $R$-module $R$ is a duo module then $R$ is right Ore. Thus for any domain $R$ which is not right Ore, the right $R$-module $R$ is a weak duo module which is not a duo module. The next result is an analogue of Proposition 1.3.

PROPOSITION 1.8. Any direct summand of a weak duo module is a weak duo module.

Proof. This is clear.

The next result is a well-known fact about direct sum decompositions that is proved for completeness.

LeMmA 1.9. Let a module $M=M_{1} \oplus M_{2}$ be a direct sum of submodules $M_{1}, M_{2}$. Then $M_{1}$ is a fully invariant submodule of $M$ if and only if $\operatorname{Hom}\left(M_{1}, M_{2}\right)=0$.

Proof. Suppose first that $M_{1}$ is a fully invariant submodule of $M$. Let $f: M_{1} \rightarrow M_{2}$ be any homomorphism. Let $p_{1}: M \rightarrow M_{1}$ denote the canonical projection and let $i_{2}: M_{2} \rightarrow M$ denote inclusion. Then $f^{*}=i_{2} f p_{1}$ is an endomorphism of $M$. By hypothesis, $f^{*}\left(M_{1}\right) \subseteq M_{1}$, so that $f\left(M_{1}\right) \subseteq M_{1} \cap M_{2}=0$. It follows that $f=0$. 
Conversely, suppose that $\operatorname{Hom}\left(M_{1}, M_{2}\right)=0$. Let $p_{2}: M \rightarrow M_{2}$ denote the canonical projection and $i_{1}: M_{1} \rightarrow M$ denote inclusion. For any endomorphism $g$ of $M$, $g\left(M_{1}\right) \subseteq p_{1} g\left(M_{1}\right)+p_{2} g i_{1}\left(M_{1}\right)=p_{1} g\left(M_{1}\right) \subseteq M_{1}$, because $p_{2} g i_{1} \in \operatorname{Hom}\left(M_{1}, M_{2}\right)=0$. It follows that $M_{1}$ is a fully invariant submodule of $M$.

Corollary 1.10. Let $M$ be a weak duo module such that $M=M_{1} \oplus M_{2}$ is a direct sum of submodules $M_{1}, M_{2}$. Then $\operatorname{Hom}\left(M_{1}, M_{2}\right)=0$.

Proof. This follows from Lemma 1.9.

A module $M$ is called Dedekind finite or directly finite if whenever $N$ is a submodule of $M$ such that $M$ is isomorphic to the module $M \oplus N$, then $N=0$. Note that the module $M$ is Dedekind finite if and only, if for any endomorphisms $f$ and $g$ of $M$ such that $f g=1$, we have $g f=1$ (see, for example, [8, Proposition 1.25]).

COROLlaRY 1.11. Weak duo modules are directly finite.

Proof. This follows from Corollary 1.10.

Generalized Hopfian modules and weakly co-Hopfian modules are directly finite. However, note that Hopfian modules need not be weak duo modules and co-Hopfian modules need not be weak duo modules. For example, let $F$ be any field and let $R$ denote the ring of upper triangular $2 \times 2$ matrices with entries in $F$. The right $R$-module $R$ is both Noetherian and Artinian and hence is both Hopfian and coHopfian. However, the right $R$-module $R$ is not a weak duo module since $R$ contains non-central idempotents.

2. Direct sums of duo modules. In this section we investigate conditions under which a direct sum of duo modules is also duo. Note that if $R$ is any ring and $M$ any non-zero $R$-module, then the $R$-module $M \oplus M$ is not a duo module. On the other hand, if $R$ is a commutative ring, $U$ and $V$ are non-isomorphic simple $R$-modules, then the $R$-module $U \oplus V$ is cyclic and hence a duo module. We begin this section with a well-known fact about direct sum decompositions that is proved for completeness

Lemma 2.1. Let a module $M=\oplus_{i \in I} M_{i}$ be a direct sum of submodules $M_{i}(i \in I)$ and let $N$ be a fully invariant submodule of $M$. Then $N=\oplus_{i \in I}\left(N \cap M_{i}\right)$.

Proof. For each $j \in I$, let $p_{j}: M \rightarrow M_{j}$ denote the canonical projection and let $i_{j}: M_{j} \rightarrow M$ denote inclusion. Then $i_{j} p_{j}$ is an endomorphism of $M$ and hence $i_{j} p_{j}(N) \subseteq$ $N$, for each $j \in I$. It follows that $N \subseteq \oplus_{j \in I} i_{j} p_{j}(N) \subseteq \bigoplus_{j \in I}\left(N \cap M_{j}\right) \subseteq N$, so that $N=$ $\oplus_{j \in I}\left(N \cap M_{j}\right)$.

A module $M$ is said to satisfy the summand sum property if $K+L$ is a direct summand of $M$ whenever $K$ and $L$ are direct summands of $M$. Also, $M$ satisfies the summand intersection property if $K \cap L$ is a direct summand of $M$ whenever $K$ and $L$ are direct summands of $M$.

COROLlary 2.2. Weak duo modules satisfy the summand sum property and the summand intersection property.

Proof. Let $K$ and $L$ be direct summands of a weak duo module $M$. There exists a submodule $L^{\prime}$ of $M$ such that $M=L \oplus L^{\prime}$. By Lemma 2.1, $K=(K \cap L) \oplus$ $\left(K \cap L^{\prime}\right)$. Thus $K \cap L$ is a direct summand of $K$ and hence also of $M$. Moreover, 
$K+L=L \oplus\left(K \cap L^{\prime}\right)$. Because $K \cap L^{\prime}$ is a direct summand of $M$ and hence also of $L^{\prime}$, we deduce that $K+L$ is a direct summand of $M$.

LeMmA 2.3. Let a module $M=\oplus_{i \in I} M_{i}$ be a direct sum of submodules $M_{i}(i \in I)$. Then the following statements are equivalent for a submodule $N$ of $M$.

(i) $N=\oplus_{i \in I}\left(N \cap M_{i}\right)$.

(ii) $N=\cap_{i \in I}\left\{N+\left(\oplus_{j \neq i} M_{j}\right)\right\}$.

Proof. (i) $\Rightarrow$ (ii). For each $i$ in $I$, let $M_{i}^{\prime}=\oplus_{j \neq i} M_{j}$. Let $i \in I$. Then $N+M_{i}^{\prime}=$ $\left\{\oplus_{j \in I}\left(N \cap M_{j}\right)\right\}+\left\{\oplus_{j \neq i} M_{j}\right\}=\left(N \cap M_{i}\right)+\left(\oplus_{j \neq i} M_{j}\right)$. It follows that $\cap_{i \in I}\left(N+M_{i}^{\prime}\right)=$ $\oplus_{i \in I}\left(N \cap M_{i}\right)=N$.

(ii) $\Rightarrow$ (i). Let $x \in N$. Then there exist a positive integer $k$, distinct elements $i_{j} \in I$ $(1 \leq j \leq k)$ and elements $m_{i} \in M_{i_{j}}(1 \leq j \leq k)$ such that $x=m_{1}+\cdots+m_{k}$. Let $1 \leq$ $j \leq k$. Clearly $m_{j} \in M_{i}^{\prime}$ for all $i \neq i_{j}$. Moreover, $m_{j}=x-m_{1}-\cdots-m_{j-1}-m_{j+1}-$ $\cdots-m_{k}$, so that $m_{j} \in N+M_{i_{j}}^{\prime}$. Thus $m_{j} \in \cap_{i \in I}\left(N+M_{i}^{\prime}\right)=N$, by (ii). It follows that $x \in\left(N \cap M_{i_{1}}\right) \oplus \cdots \oplus\left(N \cap M_{i_{k}}\right)$. Thus $N=\oplus_{i \in I}\left(N \cap M_{i}\right)$.

Let $R$ be a ring and let $M$ be a right $R$-module. For any non-empty subset $S$ of $M$, the annihilator of $S$ (in $R$ ) will be denoted by $\operatorname{ann}(S)$; i.e. $\operatorname{ann}(S)=\{r \in R: s r=0$ for all $s$ in $S\}$. In case $S=\{m\}$, then we write $\operatorname{ann}(m)$ for $\operatorname{ann}(\{m\})$. We now prove another basic fact about direct sum decompositions.

Lemma 2.4. Let a module $M=\oplus_{i \in I} M_{i}$ be a direct sum of submodules $M_{i}(i \in I)$. Then the following statements are equivalent.

(i) $R=\operatorname{ann}\left(m_{i}\right)+\operatorname{ann}\left(m_{j}\right)$ for all $m_{i} \in M_{i}, m_{j} \in M_{j}$, for all $i \neq j$ in $I$.

(ii) $N=\oplus_{i \in I}\left(N \cap M_{i}\right)$ for every (cyclic) submodule $N$ of $M$.

Moreover, in this case $\operatorname{Hom}\left(M_{i}, M_{j}\right)=0$ for all distinct $i, j$ in $I$.

Proof. (i) $\Rightarrow$ (ii). Let $N$ be any submodule of $M$. Let $m \in N$. Then there exists a positive integer $n$, distinct elements $i_{j} \in I(1 \leq j \leq n)$ and elements $m_{j} \in M_{i_{j}}(1 \leq j \leq n)$ such that $m=m_{1}+\cdots+m_{n}$. If $n=1$, then $m=m_{1} \in N \cap M_{i_{1}}$. Suppose that $n \geq 2$. By (i) there exist elements $r, s$ in $R$ such that $m_{1} r=0, m_{n} s=0$ and $1=r+s$. Then $m s=m_{1}+m_{2} s+\cdots m_{n-1} s$. Note that $m_{j} s \in M_{i_{j}}(2 \leq j \leq n-1)$ and $m s \in N$. By induction on $n, m_{1} \in N \cap M_{i_{1}}$. Similarly $m_{j} \in N \cap M_{i_{j}}(2 \leq j \leq n)$. Now (ii) follows.

(ii) $\Rightarrow$ (i). Let $i, j$ be distinct elements of $I$, let $x \in M_{i}$ and let $y \in M_{j}$. If $L=$ $(x+y) R$, then $L=\oplus_{i \in I}\left(L \cap M_{i}\right)$ and hence $x+y \in\left(L \cap M_{i}\right) \oplus\left(L \cap M_{j}\right)$. There exist $a, b \in R$ such that $x+y=(x+y) a+(x+y) b$, where $(x+y) a \in M_{i}$ and $(x+y) b \in$ $M_{j}$. Then $x=(x+y) a$, so that $x(1-a)=0$ and $y a=0$. Thus $1=(1-a)+a \in$ $\operatorname{ann}(x)+\operatorname{ann}(y)$. Now (i) follows.

Finally, let $i, j$ be distinct elements of $I$. Let $f: M_{i} \rightarrow M_{j}$ be any homomorphism. Let $n \in M_{i}$. By (i), $R=\operatorname{ann}(n)+\operatorname{ann}(f(n))$ so that $1=c+d$ for some $c, d$ in $R$ with $n c=0, f(n) d=0$. It follows that $f(n)=f(n) c+f(n) d=f(n c)+f(n) d=0$. Thus $f=0$.

Note that in Lemma 2.4, $\operatorname{Hom}\left(M_{i}, M_{j}\right)=0$ for all distinct $i, j$ in $I$ does not imply (i) (or (ii)). For let $R$ denote the ring $\mathbb{Z}$ of integers and let $M_{1}$ and $M_{2}$ denote the $R$-modules $\mathbb{Q}$ and $\mathbb{Z} / \mathbb{Z} p$, respectively, for some prime $p$. Note that $\operatorname{Hom}\left(M_{1}, M_{2}\right)=0$ and $\operatorname{Hom}\left(M_{2}, M_{1}\right)=0$. Let $N$ denote the submodule $R(1,1+\mathbb{Z} p)$ of the $R$-module $M_{1} \oplus M_{2}$. It is easy to check that if $L_{1}=M_{1} \oplus 0$ and $L_{2}=0 \oplus M_{2}$, then $N \cap L_{2}=0$ and hence $N \neq\left(N \cap L_{1}\right) \oplus\left(N \cap L_{2}\right)$. 
Let $R$ be a ring and $M$ a right $R$-module. By a complementary collection of submodules of $M$ we mean a collection of submodules $M_{i}(i \in I)$ such that $M=\oplus_{i \in I} M_{i}$. Also by a complementary pair of submodules of $M$ we mean a pair of submodules $K$, $K^{\prime}$ of $M$ such that $M=K \oplus K^{\prime}$. Thus a complementary pair of submodules of $M$ is a complementary collection of submodules of $M$ containing two submodules. Note further that $\{0, M\}$ is a complementary pair of submodules of $M$, for any module $M$.

LEMMA 2.5. The following statements are equivalent for any submodule $N$ of a module $M$.

(i) $N=\oplus_{i \in I}\left(N \cap M_{i}\right)$ for every complementary collection of submodules $M_{i}(i \in I)$ of $M$.

(ii) $N=(N \cap K) \oplus\left(N \cap K^{\prime}\right)$ for every complementary pair of submodules $K, K^{\prime}$ of $M$.

Proof. (i) $\Rightarrow$ (ii). This is clear.

(ii) $\Rightarrow$ (i). Let $M_{i}(i \in I)$ be any complementary collection of submodules of $M$. Let $x \in N$. Then there exist a positive integer $k$, distinct elements $i_{j} \in I(1 \leq j \leq k)$ and elements $m_{j} \in M_{i_{j}}(1 \leq j \leq k)$ such that $x=m_{1}+\cdots+m_{k}$. Let $1 \leq j \leq k$. By (ii), $N=\left(N \cap M_{i_{j}}\right) \oplus\left\{N \cap\left(\oplus_{i \neq i_{j}} M_{i}\right)\right\}$, so that $m_{j} \in N \cap M_{i_{j}}$. Thus $x \in \oplus_{i \in I}\left(N \cap M_{i}\right)$. It follows that $N=\oplus_{i \in I}\left(N \cap M_{i}\right)$.

COROLlary 2.6. The following statements are equivalent for a submodule $N$ of a module $M$.

(i) $N=\cap_{i \in I}\left\{N+\left(\oplus_{j \neq i} M_{j}\right)\right\}$ for every complementary collection of submodules $M_{i}$ $(i \in I)$ of $M$.

(ii) $N=(N+K) \cap\left(N+K^{\prime}\right)$ for every complementary pair of submodules $K, K^{\prime}$ of $M$.

Proof. This follows from Lemmas 2.3 and 2.5.

THEOREM 2.7. Let a module $M=\oplus_{i \in I} M_{i}$ be a direct sum of submodules $M_{i}(i \in I)$. Then $M$ is a weak duo module if and only if

(i) $M_{i}$ is a weak duo module for all $i \in I$,

(ii) $\operatorname{Hom}\left(M_{i}, M_{j}\right)=0$ for all distinct $i, j \in I$, and

(iii) $N=\oplus_{i \in I}\left(N \cap M_{i}\right)$ for every direct summand $N$ of $M$.

Proof. The necessity follows from Proposition 1.8, Corollary 1.10 and Lemma 2.1. Conversely, suppose that $M$ satisfies (i), (ii) and (iii). Let $K$ be any direct summand of $M$ and let $f$ be any endomorphism of $M$. For each $j$ in $I$ let $p_{j}: M \rightarrow M_{j}$ denote the canonical projection and let $i_{j}: M_{j} \rightarrow M$ denote inclusion. By (i), $p_{j} f i_{j}\left(K \cap M_{j}\right) \subseteq$ $K \cap M_{j}$ for all $j \in I$. Moreover, by (ii) $p_{k} f i_{j}\left(K \cap M_{j}\right)=0$ for all distinct $j, k \in I$. Now (iii) gives $f(K)=\sum_{j \in I} f\left(K \cap M_{j}\right) \subseteq \sum_{j \in I} p_{j} f i_{j}\left(K \cap M_{j}\right) \subseteq \sum_{j \in I}\left(K \cap M_{j}\right) \subseteq K$. Thus $M$ is a weak duo module.

COROllary 2.8. Let a module $M=\oplus_{i \in I} M_{i}$ be a direct sum of submodules $M_{i}$ $(i \in I)$. Then $M$ is a weak duo module if and only if

(i) $\bigoplus_{\in I^{\prime}} M_{i}$ is a weak duo module for every finite subset $I^{\prime}$ of $I$, and

(ii) $M$ satisfies the summand intersection property.

Proof. The necessity follows from Proposition 1.8 and Corollary 2.2. Conversely, suppose that $M$ satisfies (i) and (ii). In particular, note that $M_{i}$ is a weak duo module for all $i \in I$. Moreover, for all distinct elements $i, j \in I, M_{i} \oplus M_{j}$ being a weak duo module implies that $\operatorname{Hom}\left(M_{i}, M_{j}\right)=0$, by Corollary 1.10. Next let $N$ be any direct summand 
of $M$. Let $x \in N$. There exists a finite subset $I^{\prime}$ of $I$ such that $x \in \oplus_{i \in I^{\prime}} M_{i}=L$ (say). By (ii), $N \cap L$ is a direct summand of $L$. By (i) and Lemma 2.1, $N \cap L=\oplus_{i \in I^{\prime}}\left(N \cap M_{i}\right)$. It follows that $x \in \oplus_{i \in I}\left(N \cap M_{i}\right)$. Hence $N=\oplus_{i \in I}\left(N \cap M_{i}\right)$. By Theorem 2.7, $M$ is a weak duo module.

COROLlary 2.9. Let a module $M=\oplus_{i \in I} M_{i}$ be a direct sum of indecomposable submodules $M_{i}(i \in I)$. Then $M$ is a weak duo module if and only if

(i) $\operatorname{Hom}\left(M_{i}, M_{j}\right)=0$ for all distinct $i, j \in I$, and

(ii) for every direct summand $N$ of $M$ there exists a subset $I^{\prime}$ of $I$ such that $N=$ $\oplus_{i \in I^{\prime}} M_{i}$.

Proof. The necessity follows from Theorem 2.7 because weak duo modules have the summand intersection property (Corollary 2.2). The sufficiency follows by Theorem 2.7. result.

Using Lemma 2.4, the proof of Theorem 2.7 can be adapted to prove the following

THEOREM 2.10. Let a module $M=\oplus_{i \in I} M_{i}$ be a direct sum of submodules $M_{i}(i \in I)$. Then $M$ is a duo module if and only if

(i) $M_{i}$ is a duo module for all $i \in I$, and

(ii) $N=\oplus_{i \in I}\left(N \cap M_{i}\right)$ for every submodule $N$ of $M$.

Note that Lemma 2.4 shows that (ii) in Theorem 2.10 can be replaced by the equivalent condition "(ii) $R=\operatorname{ann}\left(m_{i}\right)+\operatorname{ann}\left(m_{j}\right)$ for all $m_{i} \in M_{i}, m_{j} \in M_{j}$ for all distinct elements $i, j \in I$." Compare the following result with Corollary 2.8.

COROLlary 2.11. Let a module $M=\oplus_{i \in I} M_{i}$ be a direct sum of submodules $M_{i}$ $(i \in I)$. Then $M$ is a duo module if and only if $M_{i} \oplus M_{j}$ is a duo module for all distinct $i, j$ in $I$.

Proof. The necessity follows by Proposition 1.3. Conversely, suppose that $M_{i} \oplus M_{j}$ is a duo module for all distinct $i \neq j$ in $I$. Then $M_{i}$ is a duo module for all $i \in I$, by Proposition 1.3. Moreover, for all $i \neq j$ in $I, R=\operatorname{ann}\left(m_{i}\right)+\operatorname{ann}\left(m_{j}\right)$ for all $m_{i} \in M_{i}$, $m_{j} \in M_{j}$ by Lemmas 2.1 and 2.4. Combining Lemma 2.4 and Theorem 2.10, we see that $M$ is a duo module.

3. Modules over commutative domains. Throughout this section $R$ will denote a commutative domain with field of fractions $K \neq R$. We investigate when modules over $R$ are duo modules. We begin with an elementary fact.

LEMMA 3.1. Let an R-module $M=M_{1} \oplus M_{2}$ be a direct sum of a non-zero torsionfree submodule $M_{1}$ and a non-zero submodule $M_{2}$. Then $M$ is not a duo module.

Proof. Let $m_{1}$ and $m_{2}$ be non-zero elements of $M_{1}$ and $M_{2}$, respectively. Then $\operatorname{ann}\left(m_{1}\right)=0$ and hence $\operatorname{ann}\left(m_{1}\right)+\operatorname{ann}\left(m_{2}\right)=\operatorname{ann}\left(m_{2}\right) \neq R$. By Lemma 2.4 and Theorem $2.10, M$ is not a duo module.

We first consider torsion-free modules. Note that the $R$-module $R$ is a duo module but the $R$-module $K$ is not a duo module (Proposition 1.6). Let $M$ be a torsion-free $R$-module. Without loss of generality we can suppose that $M$ is an $R$-submodule of the $K$-vector space $K \otimes_{R} M$. Let $\mathcal{O}(M)=\{k \in K: k M \subseteq M\}$. Note that $\mathcal{O}(M)$ is a subring of $K$ containing $R$. For example, if $M$ is the $R$-module $R$, then $\mathcal{O}(M)=R$. On 
the other hand, if $S$ is any subring of $K$ containing $R$ and $M$ is the $R$-module $S$, then $\mathcal{O}(M)=S$. In particular, $\mathcal{O}\left({ }_{R} K\right)=K$.

Although the next result is well known we give an elementary proof for completeness.

Lemma 3.2. Let $U$ be a torsion-free uniform $R$-module. Then a mapping $f: U \rightarrow U$ is an endomorphism of $U$ if and only if there exists $k \in \mathcal{O}(U)$ such that $f(u)=k u$ for all $u \in U$.

Proof. The sufficiency is clear. Conversely, suppose that $f$ is an endomorphism of $U$. Let $0 \neq u \in U$. Because $U$ is uniform, there exist $a, b \in R$ with $b \neq 0$ such that $b f(u)=a u$. Then $k=a / b \in K$. Let $x \in U$. There exist $c, d \in R$ with $d \neq 0$ such that $d x=c u$. Then $d f(x)=f(d x)=f(c u)=c f(u)$, so that $b d f(x)=b c f(u)=a c u=a d x$. Because $U$ is torsion-free, $b f(x)=a x$. It follows that $k x=f(x) \in U$ for all $x \in U$. Hence $k \in \mathcal{O}(U)$ and $f(x)=k x$ for all $x \in U$.

THEOREM 3.3. Let $R$ be a commutative domain. Then the following statements are equivalent for a torsion-free uniform $R$-module $U$.

(i) $U$ is a duo module.

(ii) $U$ contains a non-zero cyclic fully invariant submodule.

(iii) $\mathcal{O}(U)=R$.

Proof. (i) $\Rightarrow$ (ii) is clear.

(ii) $\Rightarrow$ (iii) Let $k \in \mathcal{O}(U)$. Then the mapping $f: U \rightarrow U$ defined by $f(u)=k u$, for all $u \in U$, is an endomorphism of $U$. Let $0 \neq x \in U$ such that $R x$ is a fully invariant submodule of $U$. Then $f(x)=r x$ for some $r \in R$ and hence $k x=f(x)=r x$, so that $k=r \in R$ because $U$ is torsion-free. It follows that $\mathcal{O}(U)=R$.

(iii) $\Rightarrow$ (i) This follows from Lemma 3.2.

In contrast to Theorem 3.3, note that any uniform $R$-module is a weak duo module.

Corollary 3.4. A commutative domain $R$ is integrally closed if and only if every finitely generated torsion-free uniform $R$-module is a duo module.

Proof. Suppose first that $R$ is integrally closed. Let $U$ be any finitely generated torsion-free uniform $R$-module. Let $k \in \mathcal{O}(U)$. Because $k U \subseteq U, k$ is integral over $R$ (see, for example, [7, Theorem 12]) and hence $k \in R$. Thus $\mathcal{O}(U)=R$. By Theorem 3.3, $U$ is a duo module. Conversely, suppose that every finitely generated torsionfree uniform $R$-module is a duo module. Let $q \in K$ such that $q$ is integral over $R$. There exist a positive integer $n$ and elements $r_{i} \in R(0 \leq i \leq n-1)$ such that $r_{0}+r_{1} q+\cdots+r_{n-1} q^{n-1}+q^{n}=0$. Let $V=R+R q+\cdots+R q^{n-1}$. Then $V$ is a finitely generated submodule of the $R$-module $K$, so that $V$ is a torsion-free uniform $R$-module. Clearly $q V \subseteq V$ so that $q \in \mathcal{O}(V)=R$, by Theorem 3.3. It follows that $R$ is integrally closed.

Note that, in general, not every torsion-free uniform $R$-module is finitely generated. Recall that an element $p$ of the domain $R$ is called prime if $R p$ is a non-zero prime ideal of $R$. Equivalently, $p$ is a non-zero non-unit of $R$ such that whenever $a, b \in R$ and $a b \in R p$ then $a \in R p$ or $b \in R p$. Recall that $R$ is a UFD if and only if every non-zero prime ideal contains a prime element (see [7, Theorem 5]). Prime elements $p$ and $q$ in $R$ are called non-associated if $R p \neq R q$. It is easy to check that prime elements $p, q$ are non-associated if and only if $R p \nsubseteq R q$ and $R q \nsubseteq R p$. 
EXAMPLE 3.5. Suppose that $R$ contains an infinite collection of non-associated prime elements $p_{i}(i \geq 1)$. Then the submodule $U=\sum_{i \geq 1} R\left(1 / p_{i}\right)$ of $K$ is a torsion-free uniform duo $R$-module that is not finitely generated.

Proof. Because it is a submodule of the $R$-module $K, U$ is a torsion-free uniform module. Let $k \in \mathcal{O}(U)$. Then $k\left(1 / p_{1}\right)=\left(a_{1} / p_{1}\right)+\cdots+\left(a_{n} / p_{n}\right)$ for some positive integer $n$ and elements $a_{i} \in R(1 \leq i \leq n)$. If $n=1$, then $k=a_{1} \in R$. Suppose that $n>$ 1. Then $k p_{2} \ldots p_{n}=b$ where $b=a_{1} p_{2} \ldots p_{n}+\cdots+a_{n} p_{1} \ldots p_{n-1} \in R$. Next $k\left(1 / p_{2}\right) \in$ $U$ so that $k\left(1 / p_{2}\right)=\left(b_{1} / p_{1}\right)+\cdots+\left(b_{t} / p_{t}\right)$ for some positive integer $t$ and elements $b_{i} \in R(1 \leq i \leq t)$. It follows that $k p_{1} p_{3} \ldots p_{n}=c \in R$. Hence $b p_{1}=k p_{1} \ldots p_{n}=c p_{2}$, so that $b \in R p_{2}$. Similarly $b \in R p_{i}(3 \leq i \leq n)$. Then $k=b /\left(p_{2} \ldots p_{n}\right) \in R$. It follows that $\mathcal{O}(U)=R$. By Theorem 3.3, $U$ is a duo module.

Finally note that $U$ is not finitely generated because $1 / p_{n+1}$ does not belong to $R\left(1 / p_{1}\right)+\cdots+R\left(1 / p_{n}\right)$ for every positive integer $n$.

Using Example 3.5 we next give an example of an $R$-module $M$ which is a direct sum $M_{1} \oplus M_{2}$ of duo modules $M_{1}$ and $M_{2}$ with $\operatorname{Hom}\left(M_{1}, M_{2}\right)=\operatorname{Hom}\left(M_{2}, M_{1}\right)=0$ but $M$ is not a duo module. (See Theorem 2.10.)

EXAMPLE 3.6. Suppose that $R$ is a UFD such that $R$ contains an infinite collection of non-associated prime elements $p_{i}(i \geq 1)$. Let $U_{1}=\sum_{i>1} R\left(1 / p_{2 i}\right)$ and $U_{2}=\sum_{i \geq 1} R\left(1 / p_{2 i-1}\right)$. Then $U_{1}$ and $U_{2}$ are both torsion-free uniform duo submodules of $K$ such that $\operatorname{Hom}\left(U_{1}, U_{2}\right)=0$ and $\operatorname{Hom}\left(U_{2}, U_{1}\right)=0$ but the $R$-module $M=$ $U_{1} \oplus U_{2}$ is not a duo module.

Proof. By Example 3.5, $U_{1}$ and $U_{2}$ are both torsion-free uniform duo submodules of $K$. Let $f: U_{1} \rightarrow U_{2}$ be any $R$-homomorphism. Because $K$ is an injective $R$-module, $f$ induces an $R$-homomorphism $g: K \rightarrow K$. Then $g(1)=k$ for some $k \in K$. It is easy to check that $f(x)=k x$ for all $x \in U_{1}$. Thus $k U_{1} \subseteq U_{2}$. Now $k\left(1 / p_{2}\right)=\left(a_{1} / p_{1}\right)+$ $\left(a_{3} / p_{3}\right)+\cdots+\left(a_{2 n-1} / p_{2 n-1}\right)$ for some positive integer $n$ and elements $a_{2 i-1} \in R(1 \leq$ $i \leq n)$. It follows that $k=b /\left(p_{1} p_{3} \ldots p_{2 n-1}\right)$ for some $b \in R$. Let $i \geq 1$. Then $k\left(1 / p_{2 i}\right) \in$ $U_{2}$ implies that $b \in R p_{2 i}$. Thus $b \in \cap_{i \geq 1} R p_{2 i}$. Because $R$ is a UFD, it follows that $b=0$. Hence $k=0$ and $f=0$. Thus $\operatorname{Hom}\left(U_{1}, U_{2}\right)=0$. Similarly $\operatorname{Hom}\left(U_{2}, U_{1}\right)=0$. Finally $M=U_{1} \oplus U_{2}$ is not a duo module by Lemma 3.1.

We now consider finitely generated torsion-free duo $R$-modules.

THEOREM 3.7. Let $R$ be a commutative domain. Then the following statements are equivalent for a non-zero finitely generated torsion-free $R$-module $M$.

(i) $M$ is a duo module.

(ii) $M$ contains a non-zero cyclic fully invariant submodule.

(iii) $M$ is a uniform module and $\mathcal{O}(M)=R$.

Proof. (i) $\Rightarrow$ (ii) is clear.

(ii) $\Rightarrow$ (iii). Let $0 \neq m \in M$ such that $R m$ is a fully invariant submodule of $M$. By Zorn's Lemma there exists a submodule $L$ of $M$ maximal such that $R m \cap L=0$. It is easy to check that $R m \oplus L$ is an essential submodule of $M$ and hence $M /(R m \oplus L)$ is a finitely generated torsion $R$-module. It follows that $a M \subseteq R m \oplus L$ for some nonzero element $a$ of $R$. Define a mapping $g: M \rightarrow R m \oplus L$ by $g(z)=a z$ for all $z \in M$. Let $u \in L$. Define a mapping $f: R m \oplus L \rightarrow M$ by $f(r m+x)=r u$ for all $r \in R, x \in L$. Then $f$ and $g$ are both homomorphisms and $f g$ is an endomorphism of $M$. Now $a u=f(a m)=f g(m) \in R m \cap L$, because $R m$ is a fully invariant submodule of $M$. Thus 
$a u=0$ and hence $u=0$. It follows that $L=0$, so that $R m$ is an essential submodule of $M$. But $R m \cong R$ which is a uniform $R$-module. Thus $M$ is uniform and $\mathcal{O}(M)=R$ by Theorem 3.3.

(iii) $\Rightarrow$ (i) This follows from Theorem 3.3.

We do not know an example of a torsion-free duo $R$-module which is not uniform. Next we prove a result involving localization. For any maximal ideal $P$ of the $\operatorname{ring} R$, $R_{P}$ will denote the localization of $R$ with respect to $P$. That is, $R_{P}$ is the subring of $K$ consisting of all elements $r / c$ with $r \in R$ and $c$ in $R \backslash P$. Let $M$ be any $R$-module. Let

$$
N=\{m \in M: c m=0 \text { for some } c \in R \backslash P\} .
$$

Then $N$ is a submodule of $M$ such that $c(m+N)=0$ for any $m \in M, c \in R \backslash P$ implies $m+N=0$. Thus we can form the localization of $\bar{M}=M / N$ with respect to $P$, which we denote by $M_{P}$. Note that the elements of $M_{P}$ are of the form $\bar{m} / c$, where $\bar{m}=$ $m+N \in \bar{M}, m \in M$ and $c \in R \backslash P$. Also note that $M_{P}$ is an $R_{P}$-module.

Proposition 3.8. Let $M$ be a module such that the $R_{P}$-module $M_{P}$ is a duo module, for every maximal ideal $P$ of $R$. Then $M$ is a duo module.

Proof. Let $m \in M$ and let $f$ be an endomorphism of $M$. Let $A=\{r \in R: r f(m) \in$ $R m\}$. Suppose that $A \neq R$. There exists a maximal ideal $P$ of $R$ such that $A \subseteq P$. Define $f^{*}: M_{P} \rightarrow M_{P}$ by $f^{*}(\bar{x} / c)=\overline{f(x)} / c$ for all $x \in M, c \in R \backslash P$. It is easy to check that $f^{*}$ is well-defined and is an endomorphism of $M_{P}$. Because $M_{P}$ is a duo $R_{P}$-module, $f^{*}(\bar{m} / 1)=(r / d)(\bar{m} / 1)$ for some $r \in R, d \in R \backslash P$. Thus $\overline{f(m)} / 1=(\overline{r m}) / d$ and it follows that $d^{\prime} f(m)=r m \in R m$ for some $d^{\prime} \in R \backslash P$. Hence $d^{\prime} \in A \subseteq P$, a contradiction. It follows that $A=R$ and hence $f(m) \in R m$. By Lemma 1.1, $M$ is a duo $R$-module.

For any module $M, E(M)$ will denote the injective envelope of $M$.

Lemma 3.9. Let $R$ be a DVR with unique maximal ideal $P=R p$ for some element $p$. Then the following statements are equivalent for a torsion $R$-module $M$.

(i) $M$ is a uniform module.

(ii) $M \cong E(R / R p)$ or $M \cong R / R p^{n}$ for some positive integer $n$.

(iii) $M$ is a duo module.

(iv) $M$ is a weak duo module.

Proof. (i) $\Rightarrow$ (ii) follows from [6, Theorem 9].

(ii) $\Rightarrow$ (iii). If (ii) holds, then $M$ is an Artinian uniserial module and hence $M$ is duo by Theorem 1.2.

(iii) $\Rightarrow$ (iv). This is clear.

(iv) $\Rightarrow$ (i). Suppose that $M$ is a weak duo module. Suppose that $X$ is a nonzero injective submodule of $M$. Then without loss of generality we can suppose that $X \cong E(R / R p)$ and that $M=X \oplus X^{\prime}$ for some submodule $X^{\prime}$. Suppose that $X^{\prime} \neq 0$. Then there exists a submodule $Y^{\prime}$ of $X^{\prime}$ such that $Y^{\prime} \cong R / R p$. Thus there exists an embedding $f: Y^{\prime} \rightarrow X$ that lifts to a non-zero homomorphism $g: X^{\prime} \rightarrow X$, because $X$ is injective. This contradicts Corollary 1.10. It follows that $X^{\prime}=0$ and hence that $M$ is uniform.

Now suppose that $M$ is reduced. By [6, Theorem 9], $M=N_{1} \oplus N_{1}^{\prime}$ for some non-zero cyclic submodule $N_{1}$ and submodule $N_{1}^{\prime}$. Suppose that $N_{1}^{\prime} \neq 0$. Again using [6, Theorem 9] we have $N_{1}^{\prime}=N_{2} \oplus N_{2}^{\prime}$ for some non-zero cyclic submodule $N_{2}$ and submodule $N_{2}^{\prime}$. For $i=1,2$, there exists a positive integer $n_{i}$ such that $N_{i} \cong R / R p^{n_{i}}$. 
Thus $\operatorname{Hom}\left(N_{1}, N_{2}\right) \neq 0$, which contradicts Corollary 1.10. It follows that $N_{1}^{\prime}=0$ and again that $M$ is uniform.

Now let $R$ be a Dedekind domain and let $M$ be an $R$-module. For each maximal ideal $P$ of $R$ let

$$
T_{P}(M)=\left\{m \in M: P^{n} m=0 \text { for some positive integer } n\right\} .
$$

Note that $T_{P}(M)$ is a submodule of $M$ and is called the P-primary component of $M$. Note further that $M$ is a torsion $R$-module if and only if $M=\oplus_{P} T_{P}(M)$, where the direct sum runs over all maximal ideals $P$ of $R$.

THEOREM 3.10. Let $R$ be a Dedekind domain. Then the following statements are equivalent for a non-zero torsion $R$-module $M$.

(i) $M$ is a duo module.

(ii) $M$ is a weak duo module.

(iii) There exist distinct maximal ideals $P_{i}(i \in I)$ of $R$ and submodules $M_{i}(i \in I)$ of $M$ such that $M=\oplus_{i \in I} M_{i}$ and, for each $i \in I$, either $M_{i} \cong E\left(R / P_{i}\right)$ or $M \cong R / P_{i}^{n_{i}}$ for some positive integer $n_{i}$.

(iv) The module $T_{P}(M)$ is zero or uniform, for every maximal ideal $P$ of $R$.

Proof. (i) $\Rightarrow$ (ii) is clear.

(ii) $\Rightarrow$ (iii). Suppose that $M$ is a weak duo $R$-module. There exist distinct maximal ideals $P_{i}(i \in I)$ of $R$ such that $T_{P_{i}}(M) \neq 0$, for all $i \in I$, and $T_{P}(M)=0$, for every, maximal ideal $P \notin\left\{P_{i}: i \in I\right\}$. Let $M_{i}=T_{P_{i}}(M)$ for each $i \in I$. Then $M=\bigoplus_{i \in I} M_{i}$. Let $i \in I$. By Proposition 1.8, $M_{i}$ is a weak duo module. It is well known that $M_{i}$ is an $R_{P_{i}}$-module and that a non-empty subset $X$ of $M_{i}$ is an $R$-submodule of $M_{i}$ if and only if $X$ is an $R_{P_{i}}$-submodule of $M_{i}$. Moreover, a mapping $f: M_{i} \rightarrow M_{i}$ is an $R$-homomorphism if and only if $f$ is an $R_{P_{i}}$-homomorphism. It follows that the $R_{P_{i}}$-module $M_{i}$ is a weak duo module. Let $S$ denote the ring $R_{P_{i}}$. By Lemma 3.9, $M_{i} \cong E\left(S / S P_{i}\right)$ or $M_{i} \cong S / S P_{i}^{n_{i}}$, for some positive integer $n_{i}$. Now [9, Proposition 5.6] gives $M_{i} \cong E\left(R / P_{i}\right)$ or $M_{i} \cong R / P_{i}^{n_{i}}$.

(iii) $\Rightarrow$ (iv). This is clear.

(iv) $\Rightarrow$ (i). Let $P$ be any maximal ideal of $R$. Then $M_{P} \cong T_{P}(M)$ as $R_{P}$-modules. By (iv), $M_{P}=0$ or $M_{P}$ is a uniform $R_{P}$-module and hence $M_{P}$ is a duo $R_{P}$-module by Lemma 3.9. Thus $M_{P}$ is a duo $R_{P}$-module for every maximal ideal $P$ of $R$. By Proposition 3.8, $M$ is a duo $R$-module.

COROLlary 3.11. Let $R$ be a Dedekind domain. Then the following statements are equivalent for a finitely generated $R$-module $M$.

(i) $M$ is a duo module.

(ii) $M$ is a weak duo module.

(iii) $M \cong I$ for some ideal I of $R$ or $M \cong\left(R / P_{1}^{n_{1}}\right) \oplus \cdots \oplus\left(R / P_{k}^{n_{k}}\right)$ for some positive integers $k, n_{1}, \ldots, n_{k}$ and distinct maximal ideals $P_{i}(1 \leq i \leq k)$ of $R$.

Proof. (i) $\Rightarrow$ (ii). This is clear.

(ii) $\Rightarrow$ (iii). Note first that $M=M_{1} \oplus M_{2}$, for some torsion-free submodule $M_{1}$ and torsion submodule $M_{2}$. If $M_{1} \neq 0$, then $M_{2}=0$, by Lemma 3.1. Thus $M$ is torsion-free or torsion. If $M$ is non-zero torsion, then $M \cong\left(R / P_{1}^{n_{1}}\right) \oplus \cdots \oplus\left(R / P_{k}^{n_{k}}\right)$, for some positive integers $k, n_{1}, \ldots, n_{k}$ and distinct maximal ideals $P_{i}(1 \leq i \leq k)$, by Theorem 3.10. Suppose that $M$ is torsion-free. Then $M \cong F \oplus A$ for some finitely 
generated free $R$-module $F$ and ideal $A$ of $R$. By Corollary 1.10, $M \cong I$ for some ideal $I$ of $R$.

(iii) $\Rightarrow$ (i). This follows from Corollary 3.4 and Theorem 3.10.

Note finally that if $R$ is a commutative domain which is not integrally closed then there exists a finitely generated uniform $R$-module $U$ that is not a duo module (Corollary 3.4) but which is a weak duo module.

\section{REFERENCES} 1974).

1. F. W. Anderson and K. R. Fuller, Rings and categories of modules (Springer-Verlag,

2. S. Dhompongsa and H. Tansee, The exchange property of modules with the finite exchange property, Comm. Algebra 31 (2003), 859-868. $755-779$.

3. Z. A. El-Bast and P. F. Smith, Multiplication modules, Comm. Algebra 16 (1988), 324-341.

4. A. Ghorbani and A. Haghany, Generalized Hopfian modules, J. Algebra 255 (2002),

5. A. Haghany and M. R. Vedadi, Modules whose injective endomorphisms are essential, J. Algebra 243 (2001), 765-779.

6. I. Kaplansky, Infinite abelian groups, University of Michigan Press (Ann Arbor, 1954).

7. I. Kaplansky, Commutative rings (Allyn and Bacon, Boston, 1970).

8. S. H. Mohamed and B. J. Müller, Continuous and discrete modules, London Math. Soc. Lecture Note Series No. 147 (Cambridge University Press, 1990).

9. D. W. Sharpe and P. Vamos, Injective modules (Cambridge University Press, 1972).

10. P. F. Smith, Multiplication modules and projective modules, Period. Math. Hungarica 29 (1994), 163-168.

11. W. W. Smith, Projective ideals of finite type, Canad. J. Math. 21 (1969), 1057-1061. 